\title{
Presencia y Biometría del Os peroneum en Individuos Chilenos: Estudio Radiológico
}

\author{
Presence and Biometry of Os peroneum in Chilean Individuals: Radiological Study
}

Ortega, $\mathbf{M}^{1}$. \& Olave, . $^{2}$

ORTEGA, M. \& OLAVE, E. Presencia y biometría del Os peroneum en individuos chilenos: Estudio radiológico. Int. J. Morphol., 37(4):1213-1219, 2019.

RESUMEN: El esqueleto está formado por 206 huesos constantes (200 huesos axiales y apendiculares más los 6 osículos del oído). Sin embargo, aparte de éstos existen otros huesos que pueden ser inconstantes, los que se denominan accesorios y sesamoideos. Basado en lo anterior, el objetivo fue identificar el os peroneum, que es uno de los huesos sesamoideos que podría estar presente en el pie humano, relacionando su presencia con el sexo, grupos etarios y dominancia, registrando también mediciones de cada hueso encontrado. Se realizó un estudio de tipo descriptivo, en donde se utilizaron radiografías de 200 pacientes, de ambos sexos, chilenos, de la IX región de La Araucanía, 50 de sexo masculino y 150 de sexo femenino, con edades entre 15 y 90 años. De los 200 pacientes estudiados, se encontraron $28(14 \%)$ con presencia de Os peroneum, 23 del sexo femenino y 5 de sexo masculino. En 16 (57,1\%)individuos se determinó que los huesos eran bilaterales. Los registros biométricos del hueso en estudio se muestran en tablas. Los datos obtenidos complementarán el conocimiento morfológico y médico acerca de este hueso en la población chilena, ya que el dolor en la zona lateral del pie puede ser causado por un espectro de etiologías, y con los datos obtenidos, caracterizar a nuestra población, aportando otra posible causa al dolor lateral de pie.

PALABRAS CLAVE: Anatomía radiológica; Pie; Huesos Sesamoideos; Os peroneum.

\section{INTRODUCCIÓN}

El esqueleto óseo está formado por 206 huesos constantes (200 huesos axiales y apendiculares más los 6 osiculos del oído). Sin embargo, existen también los huesos sesamoideos, generalmente pequeños, que se desarrollan junto a determinadas articulaciones del pie, de la mano y otros en el espesor de cierto número de tendones. Deriva su nombre de las semillas de sésamo, con las cuales se han comparado (Testut \& Latarjet, 1972).

Ellos están generalmente incrustados en los tendones íntimamente relacionados con las caras articulares o donde los tendones se angulan agudamente alrededor de superficies óseas. En ambos lugares, la cara del hueso sesamoideo relacionada con el otro hueso está cubierta por cartílago articular y desliza sobre ella. Algunos han considerado a los huesos sesamoideos como primariamente articulares, esto es, incrustados en cápsulas articulares y su asociación con los tendones como secundaria. Su función no es clara, pudiendo modificar la presión, disminuir la fricción y algunas veces, alterar la dirección de tracción del tendón, como lo hace la patela (Williams et al., 1995).
El Os peroneum (OP) es uno de los huesos sesamoideos del pie humano, éste se localiza dentro del tendón del músculo fibular largo (TFL) en la región del túnel cuboide. Según la literatura se puede encontrar hasta en el $20 \%$ de los adultos, es bilateral en el $60 \%$ de los casos. Se ha encontrado que es bipartito en el $30 \%$ de los adultos con un OP (Brigido et al., 2005).

El dolor en la zona lateral del pie puede ser causado por un espectro de etiologías como en cualquiera otra región del cuerpo; el síndrome del Os peroneum es el resultado de un espectro de afecciones que incluye uno o más de los siguientes:

- Una fractura aguda del OP o una diástasis de un OP multipartido, ocasionando una discontinuidad del TFL;

- Fractura crónica (cicatrizante) del OP o diastasis de un OP multipartido con formación de callo, las cuales pueden ocasionar una tenosinovitis estenosante del TFL;

- Desgaste o rotura parcial del TFL, proximal o distal al OP;

\footnotetext{
${ }^{1}$ Programa de Magíster en Ciencias, mención Morfología, Universidad de La Frontera, Temuco, Chile.
}

${ }^{2}$ Facultad de Medicina, Universidad de La Frontera, Temuco, Chile. 
- Ruptura del TFL con discontinuidad proximal o distal al OP; y / o

- La presencia de un tubérculo peroneo grande en la cara lateral del calcáneo que atrape el TFL y / o OP durante la excursión del tendón.

Se ha sugerido que la presencia de un OP osificado predispone a la ruptura del TFL (Sobel et al., 1994; Bashir et al., 2008; Oh et al., 2013).

El objetivo de esta investigación fue determinar la localización, prevalencia y biometría del Os peroneum en una población chilena y relacionar su presencia con la edad, sexo y predominancia (izquierda o derecha). Esta información será un gran aporte a la escasa literatura que hay de este hueso en individuos chilenos, siendo además un tema de importante aporte al ámbito morfológico y médico.

\section{MATERIAL Y MÉTODO}

En la actualidad gracias a los avances tecnológicos, específicamente con las técnicas de imagenología como la radiología convencional, tomografía axial computarizada y resonancia magnética, se pueden hacer estudios de prevalencia, biométricos y de posibles patologías que afecten a estos huesos sesamoideos.

Se realizó un estudio de tipo descriptivo, en donde se utilizaron radiografías de 200 pacientes, de ambos sexos, chilenos, de la IX región de La Araucanía, ya existentes en el sistema y que fueron solicitadas bajo criterio médico, se solicitó una dispensa del consentimiento informado al comité de ética del Servicio de Salud Araucanía Sur, debido a que no se usaron datos sensibles de los pacientes, solo se consideraron sexo, edad y la imagen radiológica sin datos, por lo que nombre, rut $\mathrm{u}$ otro dato sensible no fueron usados, con esto se aseguró el anonimato del paciente.

Mediante la técnica imagenológica de Radiografía convencional, se procedió a identificar y medir el $O s$ peroneum, específicamente en las radiografías de pies bilaterales en las proyecciones antero-posterior (AP), lateral (LAT) y oblicuas (OBL).

La recolección de los datos se realizó analizando cada una de las radiografías, en orden desde ante píe a retropié y desde medial a lateral.

Las medidas fueron realizadas en un eje longitudinal (proximal a distal del Os peroneum) y horizontal (medial a lateral del Os peroneum).
Se consideraron pacientes con los siguientes criterios:

- Pacientes chilenos.

- Pacientes mayores de 15 años.

- Pacientes en los que se hayan radiografiado ambos pies.

- Que en su examen contenga mínimo dos proyecciones, AP-OBL; AP-LAT; AP-LAT-OBL.

- Sin anatomía distorsionada.

*AP: Antero-Posterior; OBL: Oblicuo; LAT: Lateral

\section{Protocolos: Bontrager \& Klajn (2004)}

\section{Protocolo Pie AP.}

- Paciente sentado o decúbito supino en la camilla, con rodillas en semi-flexión, zona plantar de los pies apoyada en la camilla radiolúcida.

. $100 \mathrm{~cm}$ de distancia entre el equipo de rayos x y el detector.

- Haz de radiación angulado $15^{\circ}$ hacia proximal.

- Centraje por el frente, siguiendo el eje longitudinal del pie.

- Centraje por el lado, epífisis proximal del III metatarsiano

- Colimación acotada a la estructura de estudio.

\section{Protocolo Pie LAT.}

- Paciente decúbito lateral en la camilla radiolucida, borde lateral del pie apoyado en la camilla.

- Pie en lateral estricto.

. $100 \mathrm{~cm}$ de distancia entre el equipo de rayos x y el detector.

- Haz de radiación perpendicular al detector.

- Centraje por el frente, punto medio del pie en lateral.

- Centraje por el lado, epífisis proximal del III metatarsiano del pie en lateral.

. Colimación acotada a la estructura de estudio.

Protocolo Pie OBL.

- Paciente sentado o decúbito supino en la camilla radiolucida, con rodillas en semiflexion, zona plantar interna del pie apoyada sobre la camilla, zona plantar externa del pie, levantada en $30-40^{\circ}$.

$.100 \mathrm{~cm}$ de distancia entre el equipo de rayos $\mathrm{x}$ y el detector.

- Haz de radiación perpendicular al detector.

. Centraje por el frente, siguiendo el eje longitudinal del pie.

- Centraje por el lado, epífisis proximal del III metatarsiano - Colimación acotada a la estructura de estudio.

Los datos obtenidos fueron registrados en una planilla Excel, para luego ser tratados estadísticamente en el programa SPSS (versión 23 ). 


\section{RESULTADOS}

Del total de casos (200 personas), la presencia de $O s$ peroneum en borde lateral del pie, fue observada en 28 personas $(14 \%)$, de los cuales $5(17,9 \%)$ eran del sexo masculino y $23(82,1 \%)$ del femenino. Este hueso se presentó bilateralmente en 16 individuos ( 3 de sexo masculino y 13 de sexo femenino) y unilateralmente en 12 ( 2 de sexo masculino y 10 de sexo femenino).

La presencia unilateral observada en el sexo masculino fue 2 en pie derecho y 0 en el izquierdo; en el sexo femenino se presentaron 4 en el pie derecho y 6 en el pie izquierdo. Del total de personas de sexo femenino (150), el Os peroneum se encontró en $23(15,3 \%)$ y del total de sexo masculino (50), se presentó en 5 (10\%).

En relación a las variables de presencia del $O s$ peroneum y el sexo, se determinó a través de la razón de

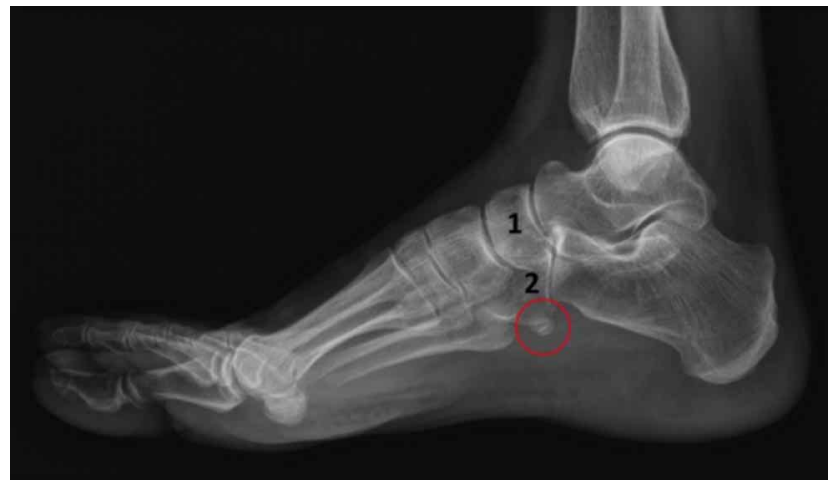

Fig. 1. Radiografía de pie derecho, sexo femenino, 78 años; 1 . Hueso navicular, 2. Hueso cuboides. Círculo rojo; Os peroneum.

verosimilitud, con un valor $\mathrm{P}>0,05$ del intervalo de confianza, que no hay una relación estadísticamente significativa entre ellas, por lo que podemos decir que la presencia de este hueso no está relacionada con el sexo de la persona.

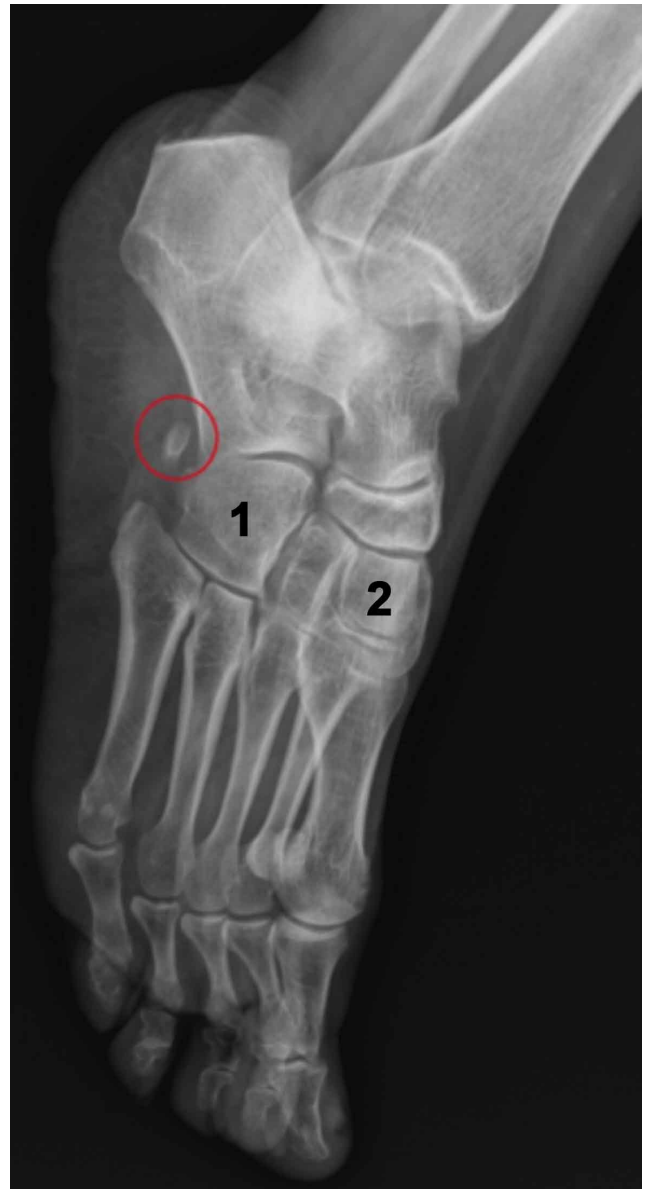

Fig. 2. Radiografía de pie izquierdo, sexo femenino, 21 años; 1 . Hueso navicular, 2. Hueso cuboides. Círculo rojo; Os peroneum.

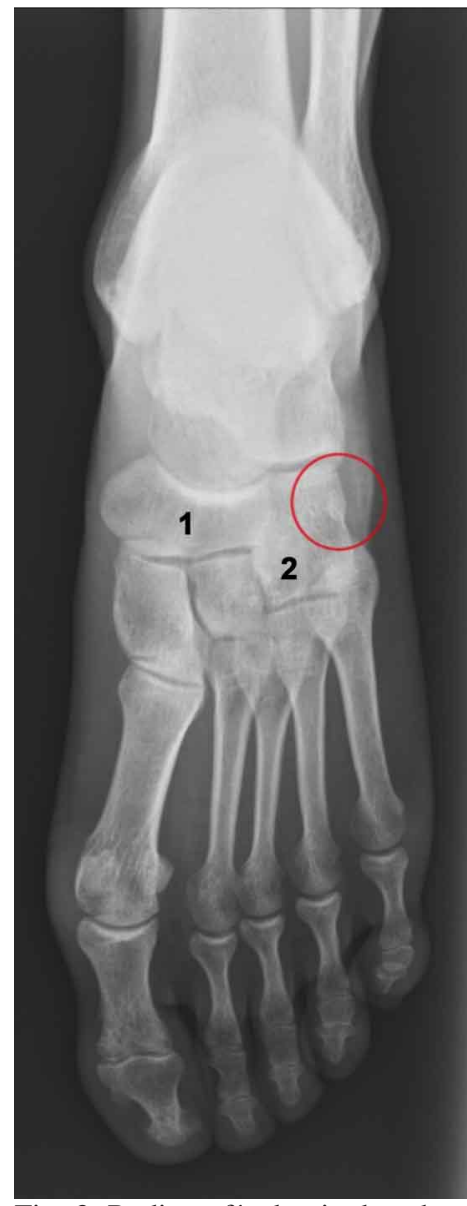

Fig. 3. Radiografía de pie derecho, sexo masculino, 73 años; 1 . Hueso navicular, 2. Hueso cuboides. Círculo rojo; Os peroneum.
La distribución del $O s$ peroneum por grupos etarios se muestra en la Tabla I.

Enfrentando las variables de presencia del Os peroneum y los grupos etarios, se determinó a través de la razón de verosimilitud; con un valor >0,05 del intervalo de confianza, que no hay una relación estadísticamente significativa entre ellas.

Los valores de longitud y ancho del Os peroneum se muestran en la Tabla II para el sexo masculino y en la Tabla III para el sexo femenino.

Mediante la prueba de normalidad de Kolmogorov-Smirnov (KS) se determinó que la distribución de los datos de la longitud del Os peroneum derecho no es normal, por lo que se utilizó la prueba no paramétrica de $\mathrm{U}$ de Mann-Whitney (U-MW), la cual determinó que la longitud del $O s$ peroneum derecho no tiene una variación estadísticamente significativa con respecto al sexo del paciente.

Por prueba KS se determinó que la distribución de la longitud del 
Tabla I. Distribución de Os peroneum por grupos etarios de la presencia de Os peroneum en individuos chilenos.

\begin{tabular}{|c|c|c|c|c|c|c|c|c|c|c|c|}
\hline \multirow{2}{*}{$\begin{array}{l}\text { Edad } \\
\text { Sexo }\end{array}$} & \multicolumn{2}{|c|}{15 a 30} & \multicolumn{2}{|c|}{31 a 45} & \multicolumn{2}{|c|}{46 a 60} & \multicolumn{2}{|c|}{61 a 75} & \multicolumn{2}{|c|}{76 a $90+$} & \multirow[t]{2}{*}{ Total } \\
\hline & $\mathrm{F}$ & M & $\mathrm{F}$ & M & $\mathrm{F}$ & M & $\mathrm{F}$ & $\mathrm{M}$ & $\mathrm{F}$ & M & \\
\hline Pie derecho & 1 & 0 & 1 & 0 & 1 & 0 & 1 & 2 & 0 & 0 & 6 \\
\hline Pie izquierdo & 0 & 0 & 1 & 0 & 3 & 0 & 2 & 0 & 0 & 0 & 6 \\
\hline Bilater al & 1 & 0 & 2 & 1 & 5 & 1 & 4 & 0 & 1 & 1 & 16 \\
\hline Total & \multicolumn{2}{|c|}{2} & \multicolumn{2}{|c|}{5} & \multicolumn{2}{|c|}{10} & \multicolumn{2}{|c|}{9} & \multicolumn{2}{|c|}{2} & 28 \\
\hline
\end{tabular}

Tabla II. Valores promedios, mínimos y máximos de las medidas de longitud y ancho de Os peroneumen en individuos chilenos de sexo masculino.

\begin{tabular}{|c|c|c|c|c|c|c|c|c|c|c|c|}
\hline \multirow{2}{*}{$\begin{array}{l}\text { Edad } \\
\text { Sexo }\end{array}$} & \multicolumn{2}{|c|}{15 a 30} & \multicolumn{2}{|c|}{31 a 45} & \multicolumn{2}{|c|}{46 a 60} & \multicolumn{2}{|c|}{61 a 75} & \multicolumn{2}{|c|}{76 a $90+$} & \multirow[t]{2}{*}{ Total } \\
\hline & $\mathrm{F}$ & M & $\mathrm{F}$ & M & $\mathrm{F}$ & M & $\mathrm{F}$ & M & $\mathrm{F}$ & M & \\
\hline Pie derecho & 1 & 0 & 1 & 0 & 1 & 0 & 1 & 2 & 0 & 0 & 6 \\
\hline Pie izquierdo & 0 & 0 & 1 & 0 & 3 & 0 & 2 & 0 & 0 & 0 & 6 \\
\hline Bilateral & 1 & 0 & 2 & 1 & 5 & 1 & 4 & 0 & 1 & 1 & 16 \\
\hline Total & & & & & & & & & & & 28 \\
\hline
\end{tabular}

Tabla III. Valores promedio, mínimos y máximos de la longitud y ancho de Os peroneumen en individuos chilenos de sexo femenino.

\begin{tabular}{lcccc}
\hline & $\begin{array}{c}\text { Longitud Os peroneum } \\
\text { derecho }(\mathrm{mm})\end{array}$ & $\begin{array}{c}\text { Longitud Os peroneum } \\
\text { izauierdo }(\mathrm{mm})\end{array}$ & $\begin{array}{c}\text { Ancho Os peroneum } \\
\text { derecho }(\mathrm{mm})\end{array}$ & $\begin{array}{c}\text { Ancho Os peroneum } \\
\text { izauierdo }(\mathrm{mm})\end{array}$ \\
\hline Media & 10,13 & 9,81 & 5,34 & 4,50 \\
DS & 3,88 & 3,56 & 2,60 & 1,74 \\
Mínimo & 3,41 & 3,22 & 1,46 & 2,80 \\
Máximo & 18,27 & 14,73 & 10,05 & 8,73 \\
\hline
\end{tabular}

Os peroneum izquierdo no es normal, la prueba no paramétrica U-MW dio como resultado que la longitud del Os peroneum izquierdo no tiene una variación estadísticamente significativa con respecto al sexo del paciente.

Por prueba KS se determinó que la distribución del ancho del Os peroneum derecho no es normal, la prueba no paramétrica U-MW dio como resultado que el ancho del $O s$ peroneum derecho no tiene una variación estadísticamente significativa con respecto al sexo del paciente.

Por prueba KS se determinó que la distribución del ancho del Os peroneum izquierdo no es normal, la prueba no paramétrica U-MW dio como resultado que el ancho del os peroneum izquierdo no tiene una variación estadísticamente significativa con respecto al sexo del paciente.

En relación a longitud y ancho del Os peroneum respecto a grupos etarios, mediante la prueba de normalidad de Kolmogorov-Smirnov (KS) se determinó que la distribución de los datos de la longitud del Os peroneum derecho no es normal, por lo que se utilizó la prueba no paramétrica de Kruskal Wallis (KW), la cual determinó que la distribución de la longitud del Os peroneum derecho es la misma entre los grupos etarios.

Por prueba KS se determinó que la distribución de los datos del ancho del Os peroneum derecho no es normal, por lo que se utilizó la prueba no paramétrica de KW, la cual determinó que la distribución del ancho del Os peroneum derecho es la misma entre los grupos etarios.

Por prueba KS se determinó que la distribución de los datos de la longitud del Os peroneum izquierdo no es normal, por lo que se utilizó la prueba no paramétrica de $\mathrm{KW}$, la cual determinó que la distribución de la longitud del Os peroneum izquierdo es la misma entre los grupos etarios.

Por prueba KS se determinó que la distribución de los datos del ancho del Os peroneum izquierdo no es normal, por lo que se utilizó la prueba no paramétrica de KW, la cual determinó que la distribución del ancho del $O s$ peroneum izquierdo es la misma entre los grupos etarios. 


\section{DISCUSIÓN}

Los huesos sesamoideos son huesos pequeños, anexos a un tendón o un ligamento. Su nombre es debido a su parecido con la forma de una semilla de sésamo, Principalmente se pueden encontrar en las manos y pies, siendo variable su aparición, número y no necesariamente bilateral (Pró, 2012). Se hallan donde éstos tendones cruzan los extremos de los huesos largos de los miembros; protegen los tendones frente a un excesivo desgaste, y a menudo modifican el ángulo de inserción tendinosa (Moore \& Dalley, 2010).

En el hombre, estos pequeños huesos, de manos y pies muestran variaciones frecuentemente y hasta hace poco, tenían poco interés médico-legal, lo que contribuyó a realizar pocos estudios sobre ellos (Bizarro, 1921; Introna et al., 1998). La incidencia de estos huesos en las extremidades superiores e inferiores está disponible en los caucásicos, pero los datos numéricos para los sitios individuales no están disponibles en grupos mixtos u otros grupos raciales e, incluso en los caucásicos, las cifras de las extremidades inferiores son escasas y vagas (Williams et al., 1995). Existen estudios que demuestran la incidencia de estos huesos en diferentes poblaciones; sin embargo, la bibliografía recoge pocos estudios que abarquen un gran número de pacientes revisados, ya que fundamentalmente se detectan asociados a otras patologías (Espejo et al., 2011). Es en parte lo que motiva nuestro estudio, buscando complementar la literatura sobre estos huesos, en específico con el Os peroneum, y con esto dar el realce que merece y ser considerado en los diagnósticos diferenciales frente al dolor de pie.

El Os peroneum, de forma ovalada, está ubicado en la parte distal del tendón peronéo largo o en el plano de la articulación calcáneo cuboidea. Es uno de los huesos sesamoídeos más frecuentes en el pie y la literatura científica lo coloca junto con el accesorio navicular y el os trigonum, como los osículos de aparición más frecuentes (Espejo et al.).

Después de un traumatismo, puede producirse una fractura de fíbula, pudiendo asociarse con un desgarro del tendón del músculo fibular largo. El reconocimiento de una fractura del Os peroneum y lesiones asociadas del TFL es importante porque el diagnóstico erróneo puede dar lugar a secuelas significativas, como la inestabilidad del tobillo y el síndrome del compartimento peroneo. El diagnóstico inicial de la fractura de Os peroneum se puede retrasar debido a un bajo índice de sospecha, síntomas inespecíficos o confusión con un Os peroneum bipartido (Brigido et al., 2005)
En la literatura revisada se han descrito dos tipos de estudios para determinar su incidencia; hablamos de los estudios anatómicos y estudios radiológicos, como el nuestro, en los cuales no se encontraron diferencias significativas entre los dos métodos.

Bizarro señaló que el Os peroneum se encontró en cinco radiografías de 100 estudiadas. En un caso fue bilateral. En todos los casos apareció como una pequeña formación ósea única. Otros autores han reportado una incidencia que varía de 14 a $26 \%$ (Mains \& Sullivan, 1973), $12,3 \%$ (Le Minor, 1987) y 10-15 \% (Sarin et al., 1999).

Sararafian (1983) informó que "el peroneo siempre está presente en una etapa no osificada, cartilaginosa o fibrocartilaginosa". En estudios radiológicos, se ha informado que la osificación del Os peroneum tiene una incidencia en individuos asintomáticos normales del $5 \%$ al 14 $\%$ y en estudios anatómicos $20 \%$, ligeramente más frecuente en varones (22\%) que en mujeres $(17 \%)$ e igualmente presente a derecha o izquierda, aunque no necesariamente bilateral (Sobel et al.; Espejo et al.). En una revisión realizada por Nwawka et al. (2013) se describió que el Os peroneum se encuentra osificado en hasta el $26 \%$ de la población.

El Os peroneum está presente en su forma completamente osificada hasta en el $20 \%$ de los adultos y es bilateral en aproximadamente el $60 \%$ de los casos. Un aspecto bipartito es un hallazgo frecuente, que ocurre en aproximadamente el $30 \%$ de los adultos con Os peroneum (Brigido et al.).

En estudio morfológico se encontró la incidencia del Os peroneum en un $28 \%$ de los casos y, bajo el punto de vista radiológico, en un $26 \%$ (Grinbaum et al., 2009).

Oyedele et al. (2006), encontraron en población sudafricana, una incidencia de este hueso en $90 \%$, siendo un $95,7 \%$ de ocurrencia bilateral. Reportaron que había una relación entre aumento de la incidencia con la edad, pero no fue significativa, encontrándose más frecuente en sujetos masculinos $(52,8 \%)$ que en los femeninos $(47,2$ $\%)$, pero esta diferencia no fue significativa.

Los resultados encontrados en nuestra investigación, respecto a la presencia del Os peroneum, concuerdan con los valores menores informados en la literatura, ya que encontramos que 28 personas poseían este hueso entre 200 pacientes estudiados, lo que corresponde a un $14 \%$. La 
presencia de este hueso en el sexo femenino se encontró en 23 casos, y en el sexo masculino en 5. Estadísticamente se determinó que la presencia del Os peroneum con respecto al sexo no es significativa $(\mathrm{P}>0,05)$, por lo que la incidencia de este hueso no depende del sexo del paciente.

Pocos estudios han descrito dominancia de este hueso, pero si algunos destacan la presencia bilateral en la mayoría de los casos (Brigido et al.; Oyedele et al.), situación que se presentó en nuestro estudio en 16 casos $(57,1 \%)$, lo que muestra que un buen porcentaje de las personas que presentan este hueso, lo tienen en ambos lados.

En cuanto a unilateralidad, reportamos que su presencia en el pie derecho se observó en 6 casos $(21,4 \%)$, así como también hubo 6 en el pie izquierdo $(21,4 \%)$, por lo que no hay una dominancia establecida.

En la literatura revisada no se encontraron registros métricos de este hueso sesamoideo, a diferencia del presente estudio, en que se obtuvo una media de la longitud del $O s$ peroneum derecho e izquierdo, en el sexo masculino, de $12,07 \pm 2,86 \mathrm{~mm}$ y $9,01 \pm 0,92 \mathrm{~mm}$, respectivamente. En tanto, la media del ancho del Os peroneum derecho e izquierdo, en el sexo masculino, fue de 5,02 $\pm 0,61 \mathrm{~mm}$ y $5,28 \pm$ $1,28 \mathrm{~mm}$, respectivamente.

Con respecto al sexo femenino, la longitud del $O s$ peroneum, tanto en el pie derecho como en el izquierdo fue de $10,13 \pm 3,88 \mathrm{~mm}$ y $9,81 \pm 3,56 \mathrm{~mm}$, respectivamente y su ancho, en el pie derecho y pie izquierdo fue de 5,34 \pm $2,60 \mathrm{~mm}$ y $4,50 \pm 1,74 \mathrm{~mm}$, respectivamente.

Los registros biométricos informados en las tablas II y III no son muy distintos entre los individuos del sexo masculino y femenino: Estadísticamente se determinó que no hay una relación significativa $(\mathrm{P}>0,05)$ entre las medidas de longitud y ancho del Os peroneum y el sexo, por lo que podemos decir que el tamaño de este hueso no depende del sexo del paciente.

Dentro de la literatura consultada tampoco se encontró datos específicos de la presencia del hueso relacionada con la edad. En nuestro estudio establecimos 5 grupos etarios, observando su presencia en cada uno de ellos, pero no se obtuvo una diferencia estadísticamente significativa ( $\mathrm{P}>0,05)$, por lo que se concluyó que la presencia de este hueso no depende de la edad del sujeto.

También relacionamos las medidas de longitud y ancho de este hueso con los grupos etarios, no encontrando una relación estadísticamente significativa $(\mathrm{P}>0,05)$, entre estas variables.
Para el dolor de pie puede haber múltiples posibles diagnósticos que buscan explicar la dolencia, pero es cierto que algún problema en los huesos sesamoideos es también una de las opciones a considerar, ya que estos huesos están presentes y también pueden sufrir los mismos trastornos que cualquier otro hueso (fracturas, artrosis, desgaste, etc.). El Os peroneum es solo uno de ellos, y este estudio nos permite señalar que su tamaño no es menor y su posición característica (lateral en el pie) puede darnos una orientación hacia éste, cuando hay una zona vecina afectada por dolor.

Esperamos que los datos obtenidos abran más el camino al estudio de los huesos sesamoideos, caracterizarlos, asociarlos a las diferentes poblaciones, determinar sus funciones específicas en el cuerpo humano y determinar así su importancia en cada paciente.

ORTEGA, M. \& OLAVE, E. Presence and biometry of $O s$ peroneum in Chilean individuals. Radiological study. Int. J. Morphol., 37(4):1213-1219, 2019.

SUMMARY: The skeleton is made up of 206 constant bones (200 bones and the 6 ossicles of the ear). However, apart from these there are other bones that can be inconstant, which are called accessories and sesamoids. Based on the above, the objective was to identify to the Os peroneum, which is one of the sesamoid bones that could be present in the human foot, relating its presence with sex, age groups and dominance, also recording measurements of each bone found. A descriptive study was carried out, in which radiographs of 200 patients of both sexes were used, Chilean, from the IX region of La Araucanía, 50 male and 150 female, aged between 15 and 90 years. Of the 200 patients studied, $28(14 \%)$ were found with the presence of peroneal bone, 23 of the female sex and 5 of the male sex. In $16(57.1 \%)$ individuals it was determined that the bones were bilateral. The biometric records of the bone under study are shown in tables. The data obtained will complement the morphological and medical knowledge about this bone in the Chilean population, since the pain in the lateral zone of the foot can be caused by a spectrum of etiologies, and with the data obtained, characterize our population, providing another possible cause to lateral standing pain.

KEY WORDS: Radiological anatomy; Foot; Sesamoid bones, Os peroneum.

\section{REFERENCIAS BIBLIOGRÁFICAS}

Bashir, W.; Lewis, S.; Cullen, N. \& Connell D. Os peroneum friction syndrome complicated by sesamoid fatigue fracture: a new radiological diagnosis? Skelet. Radiol., 38(2):181-6, 2008.

Bizarro, A. H. On sesamoid and supernumerary bones of the limbs. J. Anat. 55:256-68, 1921. 
Brigido, M.; Fessell, D.; Jacobson, J.; Widman, D.; Craig; J.; Jamadar, D. et al. Radiography and US of Os peroneum fractures and associated peroneal tendon injuries: Initial Experience. Radiology. 237(1):23541, 2005.

Bontrager, K. \& Klajn, D. Posiciones radiológicas y correlación anatómica. 5th ed. Buenos Aires, Médica Panamerica, 2004.

Espejo Antúnez, L.; Cardero Durán, M.; Caro Puértolas, B. \& Mayordomo Acevedo, R. Importancia de la presencia de Os peroneum y el síndrome de fricción en el diagnóstico diferencial de la tendinopatía peronea. Fisioterapia, 33(4):183-6, 2011

Grinbaum, C. E. A.; Abreu, A.V.; Aguiar, R. O. C.; Gasparetto, E. L. \& Koch, H. A. Radiomorphological study of the peroneus longus tendon adjacent to the cuboid bone. Radiol. Bras., 42(3):151-4, 2009.

Ho, K.; Chan, K.; Lui, T. \& Chow, Y. Tendoscopic-Assisted Repair of Complete Rupture of the Peroneus Longus Associated With Displaced Fracture of the Os peroneum —Case Report. Foot \& Ankle Int., 34(11):16004, 2013.

Introna, F. Jr.; Di Vella, G. \& Campobasso, C. P. Sex determination by discriminant analysis of patella measurements. Forensic Sci. Int., 95:3945, 1998.

Le Minor, J.M. Comparative anatomy and significance of the sesamoid bone of the peroneus longus muscle (Os peroneum). J. Anat., 151:8599, 1987.

Mains, D.B. \& Sullivan, R.C. Fracture of the os perineum. A case report. $J$. Bone Joint Surg. Am., 55:1529-530, 1973.

Moore, K. L. \& Dalley, A. D. Anatomia Orientada para a Clínica. $5^{\mathrm{a}}$ ed. Rio de Janeiro, Guanabara Koogan, 2010.

Msamati, B. \& Igbigbi, P. Radiographic appearance of sesamoid bones in the hands and feet of Malawian subjects. Clin. Anat., 14(4):248-53, 2001.

Nwawka, O.; Hayashi, D.; Diaz, L.; Goud, A.; Arndt, W.; Roemer, F.; Malguria, N. \& Guermazi, A.. Sesamoids and accessory ossicles of the foot: anatomical variability and related pathology. Insights Imaging, 4:518-93, 2013.

Oyedele, O.; Maseko, C.; Mkasi, N. \& Mashanyana, M. High incidence of the Os peroneum in a cadaver sample in Johannesburg, South Africa: Possible clinical implications? Clin. Anat., 19(7):605-10, 2006.

Pro, E. Anatomia Clínica. $1^{\mathrm{a}}$ ed. Buenos Aires, Panamericana, 2012, pag. 22,24

Sararafian, S. K. Anatomy of the foot and ankle: descriptive, topographic, functional. $2^{\text {a }}$ ed. Philadelphia, PA, J.B. Lippincott Co., 1983.

Sarin, V. K.; Erickson, G. M.; Giori, N. J.; Bergman, A.G.\& Carter, D. R. Coincident development of sesamoid bones and clues to their evolution. Anat. Rec., 257:174-80, 1999.

Sobel, M.; Pavlov, H.; Geppert, M.; Thompson, F.; DiCarlo, E. \& Davis, W. Painful Os peroneum Syndrome: A Spectrum of Conditions Responsible for Plantar Lateral Foot Pain. Foot \& Ankle Int., 15(3):11224, 1994.

Testut, L. \& Latarjet, A. Tratado de Anatomía Humana. $9^{a}$ ed. Barcelona, Salvat, 1972. Tomo I pag. 451-455

Williams, P. L.; Warwick, R.; Dyson, M. \& Bannister, L. H. Gray Anatomia. $37^{\mathrm{a}}$ ed. Rio de Janeiro, Guanabara-Koogan ,1995. v.2

\author{
Dirección para Correspondencia: \\ Dr. Enrique Olave \\ Facultad de Medicina \\ Universidad de La Frontera \\ Av. Francisco Salazar 01145 \\ Temuco \\ CHILE
}

Email: e.olave@ufrontera.cl

Recibido : 22-04-2019

Aceptado: 25-06-2019 